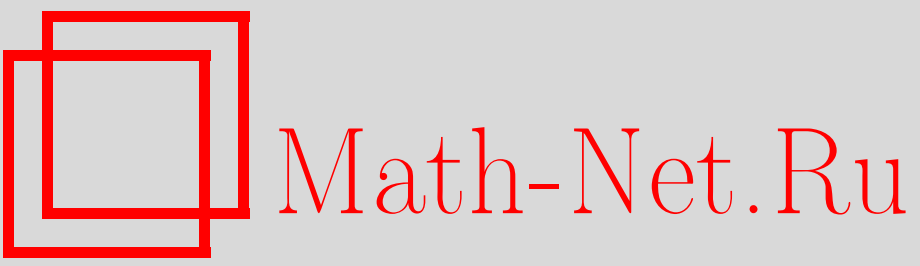

Д. А. Славнов, Квантовые измерения и колмогоровская теория вероятности, TMФ, 2003, том 136, номер 3, 436-443

DOI: https://doi.org/10.4213/tmf230

Использование Общероссийского математического портала Math-Net.Ru подразумевает, что вы прочитали и согласны с пользовательским соглашением

http://www . mathnet.ru/rus/agreement

Параметры загрузки:

IP : 107.22 .136 .117

26 апреля 2023 г., 12:56:05 
ТЕОРЕТИЧЕСКАЯ

И МАТЕМАТИЧЕСКАЯ

ФИЗИКА

Том 136, № 3

сентябрь, 2003

(C) 2003 г.

Д. А. Славнов*

\section{КВАНТОВЫЕ ИЗМЕРЕНИЯ И КОЛМОГОРОВСКАЯ ТЕОРИЯ ВЕРОЯТНОСТИ}

\footnotetext{
Установлена связь между требованием измеримости вероятностного пространства и принципом дополнительности в квантовой механике. Показано, что измеримость вероятностного пространства предполагает зависимость результатов квантового измерения не только от свойств исследуемого квантового объекта, но и от классических характеристик используемой измерительной аппаратуры. Также показано, что если учитывать требование измеримости, то в квантовом случае из гипотезы о существовании объективной реальности не следует неравенство Белла.
}

Ключевые слова: измеримость пространства, квантовое измерение, неравенство Белла.

\section{1. ВВЕДЕНИЕ}

В обзоре Хоума и Вайтейкера [1], посвяшенном интерпретации квантовой механики, есть такое высказывание: "Фундаментальная трудность в интерпретации квантовой теории состоит в том, что она в обшем случае говорит только о вероятностях получения данных результатов. Поэтому в любой дискуссии о квантовой теории многое должно определяться тем, что понимается под вероятностью - как определяется и интерпретируется этот термин". В этом обзоре довольно много места уделено описанию различных вариантов толкования понятия вероятности и лишь вскользь упомянут колмогоровский подход к проблеме вероятности [2].

Вместе с тем именно колмогоровская теория вероятности является наиболее последовательной и математически строгой. Кроме того, как представляется автору данной статьи, она наиболее полно соответствует той ситуации, которая имеет место в квантовой механике. Ниже будет описано приложение колмогоровской теории вероятности к проблеме квантовых измерений.

\section{2. ВЕРОЯТНОСТНОЕ ПРОСТРАНСТВО}

Вспомним основные положения колмогоровской теории вероятности (см., например, [2], [3]). В основе теоретико-вероятностной схемы лежит так называемое вероятностное пространство $(\Omega, \mathcal{F}, P)$. Здесь $\Omega$ - множество (пространство) элементарных событий

\footnotetext{
* Московский государственный университет, Москва, Россия. E-mail: slavnov@goa.bog.msu.ru
} 
$\omega$. Под элементарным событием понимается возможный результат одиночного эксперимента. Помимо элементарного события вводится еше просто событие, которое отождествляется с некоторым подмножеством множества $\Omega$. Считается, что в рассматриваемом эксперименте осуществляется событие $F$, если результат этого эксперимента принадлежит $F(\omega \in F)$. Совокупности подмножеств множества $\Omega$ (включающие само множество $\Omega$ и пустое множество $\varnothing$ ) наделяются структурой булевых алгебр. Алгебраическими операциями являются пересечения подмножеств, их объединение и дополнение до $\Omega$.

Вторым элементом при описании вероятностного пространства является так называемая $\sigma$-алгебра $\mathcal{F}$. Это некоторая булева алгебра, замкнутая относительно счетного числа операций объединения и пересечения. Множество $\Omega$, в котором выбрана определенная $\sigma$-алгебра $\mathcal{F}$, называется измеримым пространством. В дальнейшем измеримость будет играть ключевую роль.

Наконец, третий элемент вероятностного пространства - вероятностная мера $P$. Она представляет собой отображение алгебры $\mathcal{F}$ в множество действительных чисел, удовлетворяющее следуюшим условиям для любой счетной совокупности непересекаюшихся подмножеств $F_{j} \in \mathcal{F}$ :

а) $0 \leqslant P(F) \leqslant 1$ для всех $F \in \mathcal{F}, P(\Omega)=1$;

б) $P\left(\sum_{j} F_{j}\right)=\sum_{j} P\left(F_{j}\right)$.

Обратим внимание на то, что вероятностная мера определена только для событий, входящих в алгебру $\mathcal{F}$. Для элементарных событий вероятность, вообще говоря, не определена.

Действительной случайной величиной на $\Omega$ назьвается отображение $X$ множества $\Omega$ в расширенную действительную прямую $\bar{R}=[-\infty,+\infty]$,

$$
X(\omega)=X \in \bar{R}
$$

Предполагается, что множество $\bar{R}$ наделено свойством измеримости. В качестве $\sigma$-алгебры в множестве $\bar{R}$ можно взять булеву алгебру $\mathcal{F}_{R}$, порождаемую полуоткрытыми интервалами $\left(x_{i}, x_{j}\right]$, т.е. $\sigma$-алгебру, которая получается применением алгебраических операций к всевозможным таким интервалам. Через $\left\{\omega \in F_{R}\right\}, F_{R} \in \mathcal{F}_{R}$, обозначается подмножество элементарных событий $\omega$, для которых $X(\omega) \in F_{R}$. Подмножества $F=$ $\left\{\omega \in F_{R}\right\}$ образуют $\sigma$-алгебру $\mathcal{F}$ в пространстве $\Omega$.

\section{3. КВАНТОВЫЕ ИЗМЕРЕНИЯ}

Рассмотрим теперь приложение сформулированных основных принципов теории вероятности к проблеме квантовых измерений. Рассмотрение проведем в рамках "объективной локальной теории", восходяшей к работам Белла [4], [5], а также к статье Эйнштейна-Подольского-Розена [6].

Считается, что "объективная локальная теория" противоречит выводам стандартной квантовой механики. Далее будет предпринята попытка показать, что при использовании колмогоровской теории вероятности такое противоречие не возникает.

Будем считать, что существует некоторая объективная реальность, которая определяет возможный результат любого индивидуального измерения. Эту объективную 
реальность назовем физическим состоянием квантового объекта. Не надо путать это понятие с тем, что подразумевается под термином "состояние" в стандартной квантовой механике. Последнее будем называть квантовым состоянием. О том, какое математическое понятие можно связать с физическим состоянием, а также о соотношении между физическим и квантовым состояниями можно прочитать в статье [7].

В теории вероятности физическому состоянию сопоставим элементарное событие. Соответственно множеству физических состояний квантового объекта сопоставим пространство $\Omega$. Нужно сделать это пространство измеримым, т.е. выбрать некоторую $\sigma$-алгебру $\mathcal{F}$. Здесь решаюшее значение имеет особенность квантового измерения, которая в стандартной квантовой механике называется принципом дополнительности.

В отличие от измерений в классической физике, в квантовом случае измерения могут быть совместимыми и несовместимыми. Соответственно наблюдаемые величины в квантовой механике делятся на совместимые (одновременно измеримые) и несовместимые (дополнительные). Измерения совместимых наблюдаемых, в принципе, можно провести так, чтобы измерения одной наблюдаемой сколь угодно мало мешало проведению измерений другой наблюдаемой. Соответствующую систему измерительных приборов назовем согласованной с совокупностью этих совместимых наблюдаемых.

Для несовместимых наблюдаемых этого принципиально сделать нельзя. Как сказано в статье [8], “квантовая дополнительность просто выражает тот факт, что для того чтобы измерить две дополнительные величины, мы должны использовать приборы, которые взаимно исключают друг друга".

Таким образом, каждый индивидуальный эксперимент мы можем организовать только так, чтобы в нем измерялись совместимые наблюдаемые. Результаты измерений могут быть случайными, т.е. наблюдаемым в теории вероятности соответствуют действительные случайные величины.

Основной целью типичного квантового эксперимента является нахождение вероятностных распределений тех или иных наблюдаемых величин. При использовании определенной измерительной аппаратуры можно получить такое распределение для некоторой совокупности совместимых наблюдаемых.

С точки зрения теории вероятности, выбирая определенную измерительную аппаратуру, мы выбираем определенную $\sigma$-алгебру $\mathcal{F}$. Пусть, например, используется аппаратура, предназначенная для измерения импульса частицы. Пусть на этой аппаратуре мы можем установить, что импульс частицы лежит в интервале $\left(p_{i}, p_{j}\right]$. Для определенности мы взяли полуоткрытый интервал, хотя это и не обязательно. Попадание импульса частицы в тот или иной интервал является событием для выбранной измерительной аппаратуры. Эти события будут элементами определенной $\sigma$-алгебры.

Таким образом, вероятностное пространство $(\Omega, \mathcal{F}, P)$ определяется не только исследуемым квантовым объектом (множеством его физических состояний), но и той измерительной аппаратурой, которая при этом используется.

Предположим, проводится типичный квантовый эксперимент. Имеется ансамбль квантовых систем, находяшихся в определенном квантовом состоянии, например, частицы со спином $1 / 2$ и с проекцией спина на ось $x$, равной $1 / 2$. Пусть мы хотим исследовать распределение двух несовместимых наблюдаемых, например, проекций спина на направления, составляюшие с осью $x$ углы $\theta_{1}$ и $\theta_{2}$. В одном эксперименте обе инте- 
ресуюшие нас наблюдаемые измерить невозможно. Поэтому необходимо провести две группы экспериментов с использованием разной измерительной аппаратуры. Под словом "разной” следует понимать классически различимой. В нашем конкретном случае аппаратура должна быть различным образом ориентирована в пространстве.

Одну группу экспериментов можно описать с помошью вероятностного пространства $\left(\Omega, \mathcal{F}_{1}, P_{1}\right)$, другую - с помощью $\left(\Omega, \mathcal{F}_{2}, P_{2}\right)$. Хотя в обоих случаях пространство элементарных событий $\Omega$ одно и то же, вероятностные пространства разные. Для придания этим пространствам свойства измеримости в них введены разные $\sigma$-алгебры $\mathcal{F}_{1}$ и $\mathcal{F}_{2}$.

Формально, чисто математически, можно построить $\sigma$-алгебру $\mathcal{F}_{12}$, которая включает в себя как алгебру $\mathcal{F}_{1}$, так и алгебру $\mathcal{F}_{2}$. Такая алгебра называется порожденной алгебрами $\mathcal{F}_{1}$ и $\mathcal{F}_{2}$. Она содержит помимо подмножеств $F_{i}^{(1)} \in \mathcal{F}_{1}$ и $F_{j}^{(2)} \in \mathcal{F}_{2}$ множества $\Omega$ также всевозможные пересечения и объединения подмножеств $F_{i}^{(1)} \in \mathcal{F}_{1}$ и $F_{j}^{(2)} \in \mathcal{F}_{2}$.

Однако с физической точки зрения такая $\sigma$-алгебра будет неприемлемой. Действительно, событие $F_{i j}=F_{i}^{(1)} \cap F_{j}^{(2)}$ состоит в том, что для одного квантового объекта значения двух несовместимых наблюдаемых лежат в строго определенной области. Для квантовой системы принципиально невозможно поставить эксперимент, который смог бы выделить такое событие. Поэтому для такого события не сушествует понятия “вероятность". Другими словами, подмножеству $F_{i j}$ не соответствует никакая вероятностная мера, и $\sigma$-алгебра $\mathcal{F}_{12}$ не годится для построения вероятностного пространства. Здесь проявляется принципиальная особенность приложения теории вероятности к квантовым системам - не всякая математически возможная $\sigma$-алгебра является физически допустимой.

Как же вероятностное пространство реализуется в квантовом эксперименте? Определение вероятности предполагает многократное осушествление испытаний. Эти испытания должны проводиться в одинаковых условиях. Это относится как к испытуемому объекту, так и к измерительной аппаратуре. Ясно, что микросостояния ни того, ни другого полностью контролировать невозможно. Поэтому под словами “одинаковые условия" следует понимать некоторые классы эквивалентности состояний квантового объекта и измерительной аппаратуры.

Контролю поддаются только классические характеристики физической системы. Поэтому, фиксируя некоторые классические характеристики физической системы, мы фиксируем соответствующий класс эквивалентности.

Для исследуемого квантового объекта такой фиксацией обычно является выбор определенного квантового состояния. Например, для частиц со спином - это отбор частиц с определенной ориентацией спина. Для измерительной аппаратуры мы также должны выбрать определенную классическую характеристику, по которой фиксируется некоторый класс эквивалентности. Например, в измерительной аппаратуре первоначальный единый пучок частиц должен расщепляться на несколько достаточно хорошо отделенных друг от друга пучков, соответствующих разным значениям проекции спина на некоторое выделенное направление.

Таким образом, элементу измеримого пространства $(\Omega, \mathcal{F})$ в эксперименте соответствует совокупность квантовых объектов (например, находяшихся в определенном квантовом состоянии) и определенного типа измерительной аппаратуры, позволяющего зафиксировать событие определенного вида. Каждый такой измерительный прибор мо- 
жет выделить события, соответствующие некоторому набору совместимых наблюдаемых величин. Поэтому в квантовом случае измерительные приборы следует разделить на различные типы, соответствующие отдельной совокупности совместимых наблюдаемых. Существование различных типов измерительной аппаратуры приводит к еще одной особенности квантовых измерений. Дело в том, что одна и та же величина может входить в две (или более) различные совокупности совместимых наблюдаемых. Выделенная нами наблюдаемая совместима со всеми наблюдаемыми, входяшими в различные совокупности. Однако другие наблюдаемые из разных совокупностей не совместимы между собой. Для измерения выделенной наблюдаемой мы можем использовать различные типы измерительной аппаратуры. Это значит, что мы можем провести эксперимент в различных условиях. Нет никакой гарантии, что результат измерения не будет зависеть от этих условий.

Таким образом, результат индивидуального квантового измерения может зависеть не только от внутренних свойств измеряемого объекта (физического состояния), но и от типа измерительного прибора. В терминах теории вероятности это выражается следующим образом: для квантовой системы случайная величина $X$ может быть многозначной функцией элементарного события $\omega$.

В классическом случае все наблюдаемые совместимы между собой. Соответственно все измерительные приборы принадлежат одному типу, поэтому классическая случайная величина $X$ является однозначной функцией $\omega$. Заметим, что если в квантовом случае величину $X$ рассматривать как функцию не на пространстве $\Omega$, а на измеримом пространстве $(\Omega, \mathcal{F})$, то эта функция будет уже однозначной.

В свете сказанного следует по-новому взглянуть на результат, полученный в статье [9], где доказана запрещающая теорема. Смысл этой теоремы заключается в том, что для частицы со спином 1 не существует такой внутренней характеристики, которая однозначно предопределяет значения квадратов проекций спина на три взаимно ортогональные направления.

В стандартной квантовой механике такие три квадрата описываются взаимно коммутирующими операторами. Поэтому соответствующие наблюдаемые $\left(\widehat{S}_{x}^{2}, \widehat{S}_{y}^{2}, \widehat{S}_{z}^{2}\right)$ являются совместимыми. Совместимыми являются также наблюдаемые $\left(\widehat{S}_{x}^{2}, \widehat{S}_{y^{\prime}}^{2}, \widehat{S}_{z^{\prime}}^{2}\right)$, где направления $x, y^{\prime}, z^{\prime}$ также ортогональны между собой, но направления $y, z$ не параллельны направлениям $y^{\prime}, z^{\prime}$. Наблюдаемые $\left(\widehat{S}_{y}^{2}, \widehat{S}_{z}^{2}\right)$ не совместимы с наблюдаемыми $\left(\widehat{S}_{y^{\prime}}^{2}, \widehat{S}_{z^{\prime}}^{2}\right)$. Приборы, согласованные с наблюдаемыми $\left(\widehat{S}_{x}^{2}, \widehat{S}_{y}^{2}, \widehat{S}_{z}^{2}\right)$ и $\left(\widehat{S}_{x}^{2}, \widehat{S}_{y^{\prime}}^{2}, \widehat{S}_{z^{\prime}}^{2}\right)$, относятся к разным типам. Поэтому эти приборы не обязательно должны давать один и тот же результат при измерении квадрата проекции спина на направление $x$.

Так как в одном эксперименте невозможно одновременно использовать два типа измерительных приборов, то прямой эксперимент по проверке этого утверждения провести нельзя. Однако можно попытаться провести косвенное измерение.

Такой эксперимент, например, можно организовать следуюшим образом. Взять физическую систему, находящуюся в синглетном состоянии, которая распадается на две массивные частишы со спином 1 . У одной родившейся частишы измерить $\widehat{S}_{x}^{2}$ с помошью аппаратуры, согласованной с наблюдаемыми $\left(\widehat{S}_{x}^{2}, \widehat{S}_{y}^{2}, \widehat{S}_{z}^{2}\right)$, а у другой измерить $\widehat{S}_{x}^{2}$ с помошью аппаратуры, согласованной с наблюдаемыми $\left(\widehat{S}_{x}^{2}, \widehat{S}_{y^{\prime}}^{2}, \widehat{S}_{z^{\prime}}^{2}\right)$. После этого в каждом индивидуальном эксперименте следует сравнить результаты, получившиеся для 
каждой частицы. При этом надо тщательно следить за тем, чтобы направление $x$ не было выделенным при распаде синглетного состояния. В противном случае меж ду проекциями спина на направление $x$ для обеих частиц может иметь место дополнительная коррелящия, которая нарушит чистоту эксперимента.

Если результат измерения квадрата проекции спина на выделенную ось действительно зависит от типа используемой аппаратуры, то основное условие запрещающей теоремы Кочена и Шпекера окажется невыполненным. В таком случае эта теорема не может использоваться в качестве аргумента в пользу отсутствия объективной реальности.

\section{4. НЕРАВЕНСТВО БЕЛЛА}

Посмотрим теперь, как условие измеримости вероятностного пространства проявляет себя в таком важном случае, как вывод неравенства Белла [4]. Существует много вариантов этого неравенства. Мы будем ориентироваться на вариант, предложенный в работе [10].

Пусть частица со спином 0 распадается на две частицы $A$ и $B$ со спинами $1 / 2$. Эти частицы разлетаются на большое расстояние, и проекции их спинов измеряются двумя независимыми приборами $D_{a}$ и $D_{b}$. Пусть прибор $D_{a}$ у частицы $A$ измеряет проекцию спина на направление $a$, а прибор $D_{b}$ измеряет у частицы $B$ проекцию спина на направление $b$. Соответствуюшие наблюдаемые обозначим $\hat{A}_{a}$ и $\widehat{B}_{b}$, а результаты измерений $A_{a}$ и $B_{b}$.

Предположим, что у исходной частицы существует некоторая физическая реальность, которая может быть отмечена параметром $\lambda$. Этот же параметр будем использовать для описания физических реальностей продуктов распада. Соответственно результаты измерения наблюдаемых $\hat{A}_{a}, \widehat{B}_{b}$ можно рассматривать как функции $A_{a}(\lambda), B_{b}(\lambda)$.

Пусть распределение событий по параметру $\lambda$ характеризуется вероятностной мерой $P(\lambda)$,

$$
\int d P(\lambda)=1, \quad 0 \leqslant P(\lambda) \leqslant 1
$$

Введем корреляционную функцию

$$
E(a, b)=\int d P(\lambda) A_{a}(\lambda) B_{b}(\lambda)
$$

и рассмотрим комбинацию

$$
\begin{aligned}
I & =\left|E(a, b)-E\left(a, b^{\prime}\right)\right|+\left|E\left(a^{\prime}, b\right)+E\left(a^{\prime}, b^{\prime}\right)\right|= \\
& =\left|\int d P(\lambda) A_{a}(\lambda)\left[B_{b}(\lambda)-B_{b^{\prime}}(\lambda)\right]\right|+\left|\int d P(\lambda) A_{a^{\prime}}(\lambda)\left[B_{b}(\lambda)+B_{b^{\prime}}(\lambda)\right]\right| .
\end{aligned}
$$

При любых направлениях $a$ и $b$ выполнены равенства

$$
A_{a}(\lambda)= \pm \frac{1}{2}, \quad B_{b}(\lambda)= \pm \frac{1}{2} .
$$

Поэтому

$$
\begin{aligned}
I & \leqslant \int d P(\lambda)\left[\left|A_{a}(\lambda)\right|\left|B_{b}(\lambda)-B_{b^{\prime}}(\lambda)\right|+\left|A_{a^{\prime}}(\lambda)\right|\left|B_{b}(\lambda)+B_{b^{\prime}}(\lambda)\right|\right]= \\
& =\frac{1}{2} \int d P(\lambda)\left[\left|B_{b}(\lambda)-B_{b^{\prime}}(\lambda)\right|+\left|B_{b}(\lambda)+B_{b^{\prime}}(\lambda)\right|\right] .
\end{aligned}
$$


Согласно равенствам (3) при любом $\lambda$ одно из выражений

$$
\left|B_{b}(\lambda)-B_{b^{\prime}}(\lambda)\right|, \quad\left|B_{b}(\lambda)+B_{b^{\prime}}(\lambda)\right|
$$

равно нулю, а другое единище. Отсюда получается неравенство Белла

$$
I \leqslant \frac{1}{2} \int d P(\lambda)=\frac{1}{2}
$$

Квантово-механическое вычисление для корреляционной функции дает результат

$$
E(a, b)=-\frac{1}{4} \cos \theta_{a b},
$$

где $\theta_{a b}$ - угол между направлениями $a$ и $b$. При направлениях $a=0, b=\pi / 8, a^{\prime}=\pi / 4$, $b^{\prime}=3 \pi / 8$ для $I$ получаем

$$
I=\frac{1}{\sqrt{2}}
$$

что противоречит неравенству (5).

Проведенные эксперименты соответствуют квантово-механическим расчетам и не подтверждают неравенства Белла. Эти результаты рассматриваются как решаюшие свидетельства против гипотезы о существовании объективной локальной реальности в квантовой физике. Легко убедиться, что если учитывать особенность приложения теории вероятности к квантовым системам, то подобный вывод неравенства Белла провести нельзя. Так как в квантовом случае $\sigma$-алгебра и соответственно вероятностная мера зависят от используемой измерительной аппаратуры, то формулу (1) следует переписать в виде

$$
E(a, b)=\int d P_{a b}(\omega) A_{a}(\omega) B_{b}(\omega) .
$$

Формула (2) теперь будет выглядеть так:

$$
\begin{aligned}
I= & \left|\int d P_{a b}(\omega) A_{a}(\omega) B_{b}(\omega)-\int d P_{a b^{\prime}}(\omega) A_{a}(\omega) B_{b^{\prime}}(\omega)\right|+ \\
& +\left|\int d P_{a^{\prime} b}(\omega) A_{a^{\prime}}(\omega) B_{b}(\omega)+\int d P_{a^{\prime} b^{\prime}}(\omega) A_{a^{\prime}}(\omega) B_{b^{\prime}}(\omega)\right| .
\end{aligned}
$$

Если направления $a$ и $a^{\prime}\left(b\right.$ и $\left.b^{\prime}\right)$ не параллельны друг другу, то наблюдаемые $\hat{A}_{a} \widehat{B}_{b}$, $\hat{A}_{a} \widehat{B}_{b^{\prime}}, \hat{A}_{a^{\prime}} \widehat{B}_{b}, \hat{A}_{a^{\prime}} \widehat{B}_{b^{\prime}}$ являются взаимно несовместимыми. Поэтому не существует единой физически допустимой $\sigma$-алгебры, которая соответствовала бы измерению всех этих наблюдаемых. Отсюда следует, что нет единой вероятностной меры для этих наблюдаемых. Соответственно четыре интеграла, которые фигурируют в правой части формулы (6), объединить в один интеграл нельзя. В результате переход к неравенству типа (4) оказывается невозможным.

Таким образом, гипотеза о существовании локальной объективной реальности в квантовом случае не ведет к неравенствам Белла. Поэтому многочисленные экспериментальные проверки неравенств Белла, которые проводились ранее и проводятся сейчас, в большой степени лишаются теоретической базы. Конечно, если бы удалось получить экспериментальное подтверждение существования неравенств Белла, то это была бы сенсация. Однако отрицательный результат ничего не доказьвает. 


\section{5. ЗАКЛЮЧЕНИЕ}

Большинство экспериментальных исследований дилеммы - существует или не существует локальная объективная реальность в квантовой физике, сводится к проверке наличия или отсутствия интерференционной картины в том или ином случае. С некоторыми примерами подобного рода можно познакомиться в цитированной ранее статье [8]. Ясно, что интерференционная картина определяется вероятностным распределением, характеризуюшим рассматриваемый физический процесс. В свою очередь, как показано выше, вероятностное распределение в большой степени определяется типом измерительной аппаратуры, которая используется в данном наблюдении.

В таком контексте выглядят вполне естественными описанные в кратком обзоре в статье [8] результаты Допфера, который практически осушествил эксперимент, аналогичный мысленному эксперименту с так называемым микроскопом Гайзенберга. В этом эксперименте наличие или отсутствие интерференционной картины зависит от того, на каком расстоянии от линзы помешается детектор.

Из всего сказанного выше следует, что концепция локальной объективной реальности и восходящий к Бору “ситуационный (контекстуальный) подход" не находятся в таком антагонистическом противоречии, как это принято считать. Вполне допустимо, что сушествует физическая реальность, присушая исследуемому квантовому объекту, которая предопределяет результат любого эксперимента. Однако этот результат может зависеть от условий, в которых проводится эксперимент. В число этих условий входит классическая характеристика (тип) измерительной аппаратуры, используемой в конкретном случае.

\section{Список литературы}

[1] D. Home, M. A. B. Whitaker. Phys. Rep. 1992. V. 210. P. 223.

[2] А.Н. Колмогоров. Основные понятия теории вероятности. М.: Наука, 1974.

[3] Ж. Неве. Математические основы теории вероятности. М.: Мир, 1969.

[4] J.S. Bell. Physics. 1965. V. 1. P. 195.

[5] J.S. Bell. Speakable and Unspeakable in Quantom Mechanics. Cambridge: Cambridge University Press, 1987.

[6] А. Эйнштейн, Б. Подольский, Н. Розен. УФН. 1936. Т. 16. С. 440.

[7] Д. А. Славнов. ТМФ. 2001. Т. 132. С. 434.

[8] A. Zeilinger. Rev. Modern. Phys. 1999. V. 71. P. S289.

[9] S. Kochen, E. P. Specker. J. Math. Mech. 1967. V. 17. P. 59.

[10] J. F. Clauser, M. A. Horn, A. Shimony, R. A. Holt. Phys. Rev. Lett. 1969. V. 23. P. 880. 